\title{
FASN and CD36 predict survival in rituximab-treated diffuse large B-cell lymphoma
}

\author{
Olga V. Danilova • Larry J. Dumont • Norman B. Levy • \\ Frederick Lansigan • William B. Kinlaw • \\ Alexey V. Danilov $\cdot$ Prabhjot Kaur
}

Received: 19 June 2012 / Accepted: 7 September 2012 / Published online: 5 October 2012

(C) Springer-Verlag 2012

\begin{abstract}
Diffuse large B-cell lymphoma is the most common lymphoid malignancy, as it accounts for approximately one third of all patient cases of non-Hodgkin's lymphoma. Patients with diffuse large B-cell lymphoma have markedly different treatment outcomes, suggesting a need for reliable prognostic factors and novel therapeutic approaches. De novo fatty acid synthesis is an important metabolic driver of tumor in multiple malignancies. In this retrospective study, we analyzed expression of fatty acid synthase (a key enzyme in de novo fatty acid synthesis), Spot 14 (thyroid hormone responsive Spot 14, a nuclear protein that promotes expression of genes involved in fatty acid synthesis), and CD36 (the cell surface channel for exogenous fatty acid uptake) in patients with diffuse large B-cell lymphoma and their clinical significance. We observed that overexpression of fatty acid synthase is negatively associated with overall survival $(p=0.001)$ and progression-free period $(p=0.004)$ in patients with diffuse large B-cell lymphoma. Multivariate analysis showed that fatty acid synthase overexpression is
\end{abstract}

Electronic supplementary material The online version of this article (doi:10.1007/s12308-012-0166-4) contains supplementary material, which is available to authorized users.

O. V. Danilova $\cdot$ L. J. Dumont $\cdot$ N. B. Levy $\cdot$ P. Kaur $(\bowtie)$

Department of Pathology, Dartmouth-Hitchcock Medical Center, Lebanon, NH 03756, USA

e-mail: prabhjot.kaur@hitchcock.org

F. Lansigan · W. B. Kinlaw · A. V. Danilov

Department of Medicine, Dartmouth-Hitchcock Medical Center, Lebanon, NH, USA

L. J. Dumont • N. B. Levy · F. Lansigan · W. B. Kinlaw •

A. V. Danilov $\cdot$ P. Kaur

Geisel School of Medicine at Dartmouth,

Hanover, NH, USA

N. B. Levy · F. Lansigan - W. B. Kinlaw $\cdot$ A. V. Danilov $\cdot$ P. Kaur Norris Cotton Cancer Center,

Lebanon, NH, USA an independent prognostic marker of aggressive clinical course. For the first time, we report CD36 as an independent protective factor in patients treated with rituximab. Thus, fatty acid synthase and CD36 expression may serve as prognostic markers to predict response to treatment and survival in diffuse large B-cell lymphoma patients. Fatty acid synthase may also be a potential therapeutic target in lymphoid malignancies.

Keywords Fatty acid synthase $\cdot$ CD36 $\cdot$ S14 $\cdot$ Lipogenesis · Survival · Diffuse large B-cell lymphoma

\section{Introduction}

Diffuse large B-cell lymphoma (DLBCL) is the most common malignant lymphoma in the Western world and accounts for 35-40\% of all cases of non-Hodgkin's lymphoma diagnosed in the USA each year [1]. The biology and pathogenesis of DLBCL remain elusive, and up to $50 \%$ of the patients succumb to their illness despite anthracyclinebased multiagent chemotherapy [2]. Thus, search for reliable prognostic factors in DLBCL in an attempt to identify patients who may benefit from a more aggressive treatment strategy and development of new therapeutic approaches remain an unmet clinical need.

Most aggressive tumors demonstrate increased glucose uptake and trapping. Increased glycolysis in the presence of oxygen (the Warburg effect) and high rates of protein and DNA synthesis are well-described features of the transformed phenotype [3-7]. The role of de novo fatty acid (FA) synthesis in carcinogenesis, initially described in the 1950s [8], has recently received increased investigative attention $[9,10]$. Multifunctional, homodimeric FASN catalyzes de novo biosynthesis of endogenous FAs, which are essential constituents of cell membrane lipids and important 
substrates for energy production [11, 12]. In normal tissues of individuals consuming a Western diet rich in FA, FASN expression and the rate of de novo synthesis are low. In contrast, FASN is overexpressed in a variety of malignancies including leukemia and multiple myeloma $[13,14]$ and has been shown to correlate with tumor progression, aggressiveness, and metastasis in tumors of the prostate, breast, ovary, lung, and colorectum $[10,15-22,10,22]$. FASN inhibition also represents a potential therapeutic approach $[9,21,23,24]$.

Spot 14 [S14; thyroid hormone responsive Spot 14 (THRSP)], is primarily a nuclear protein [25] and is suggested to regulate gene transcription of FA synthesis enzymes. S14 messenger RNA is expressed abundantly in lactating mammary gland, most breast-cancer-derived cell lines, and $\sim 75 \%$ of breast cancer specimens, which are major sites of active lipogenesis [26, 27]. The human S14 gene is located at 11q13.5, a region that is amplified in a subset of aggressive breast cancers. In tumors of the breast, S14 expression correlates with disease prognosis [28, 27, 29]. Inhibition of S14 expression in rat hepatocytes and cancer cells prevents activation of genes encoding the enzymes of FA synthesis, including fatty acid synthase (FASN), acetyl CoA-carboxylase, and ATP citrate lyase. [30, 31-33]. Evaluation of S14 expression as a prognostic factor in clinical DLBCL has not been reported previously.

CD36 is a multiligand receptor first identified on platelets as glycoprotein IV, an 88-kDa thrombospondin and collagen receptor, and was linked to lipid metabolism as a macrophage receptor for oxidized low-density lipoprotein and as an adipocyte receptor/transporter for long-chain FAs [34, 35]. CD36 is a cell surface class $B$ scavenger receptor, which localizes to the lipid rafts to facilitate their association with receptors, signaling, and adapter molecules [36]. CD36 is expressed in neoplastic cells where it binds, promotes internalization, and regulates transport of long-chain FAs $[37,34,38]$. CD38 expression has been explored in de novo CD5-positive DLBCL and CLL [39, 40], but the precise functional role of CD36 in DLBCL remains unclear. In this study, we analyzed the expression of FASN, S14, and CD36 in DLBCL and the potential clinical significance.

\section{Methods}

Patients and samples Consecutive cases of DLBCL ( $n=$ 125) were identified from the files of Department of Pathology at Dartmouth-Hitchcock Medical Center (diagnosed between 1996 and 2008). Eighty-six evaluable cases DLBCL, NOS, were selected for the study and comprised all cases that did not belong to specific subtypes [2]. Cases that belonged to specific subtypes (e.g., T cell/histiocyterich diffuse large B-cell lymphoma, primary central nervous system lymphoma), or borderline cases (e.g., B-cell lymphoma, unclassifiable with features intermediate between DLBCL and Burkitt lymphoma), lacked adequate paraffin embedded tissue, sufficient follow-up period, and essential clinical data were excluded. Burkitt and Burkitt lymphoma variants were excluded based on the World Health Organization criteria to include morphology, Ki-67 index, and cytogenetics. The extent of the disease at presentation was determined through physical examination, serum lactate dehydrogenase, complete blood count, bone marrow aspirate and biopsy, chest X-ray, and computed tomography of the chest, abdomen, and pelvis, or positron emission tomography scan. Age at diagnosis, gender, Ann Arbor stage at presentation, performance status, International Prognostic Index (IPI), the revised IPI for those treated with rituximab $[41,42]$, and chemotherapy were extracted from medical records. IPI and revised IPI (R-IPI) were categorized into low risk (scores $0-2$ ) and high risk (scores 3-5). In the rituximab, cyclophosphamide, doxorubicin, vincristine, and prednisone (R-CHOP) patients, we found that the assignments to low- or high-risk categories were identical using IPI and R-IPI scores. Therefore, we use IPI to designate this variable throughout this report. Primary outcomes were overall survival (time from diagnosis to the endpoint), progression-free survival (time from diagnosis until progression or death), and number of treatment regimens required to achieve a remission (no evidence of clinical disease and/ or by imaging for at least 6 months after completion of therapy). The study was closed to follow-up on April 15, 2011. The institutional review board of the DartmouthHitchcock Medical Center approved the study.

Immunohistochemistry and construction of tissue microarray Tissue microarrays (TMAs) containing the 86 cases of DLBCL were analyzed by immunohistochemistry (IHC) for CD10; Bc16; MUM1; FASN with an affinity-purified rabbit antihuman FASN immunoglobulin $\mathrm{G}(\mathrm{IgG})$ preparation (Abcam, MA, USA), dilution 1:100; S14 with a validated, purified mouse monoclonal antibody K/IIIC5.1, an IgG type 2a [33]; and CD36 (PA1-46480,Thermo Scientific, USA), dilution 1:100. On an average, two (one to four) $1.0 \mathrm{~mm}$ diameter tissue cylinders were punched from archival formalin-fixed, paraffin-embedded blocks and transferred into a fresh paraffin block. Four micrometer sections were cut from each TMA and submitted for hematoxylin and eosin staining and IHC. Tissue sections were mounted onto charged Biogenix Plus slides (San Ramon, CA, USA), deparaffinized, and rehydrated through graded alcohols before immunostaining. All immunohistochemical staining was performed after antigen retrieval using intermittent heating for 4 cycles of 5 min each in a $625-\mathrm{W}$ microwave oven to maintain the temperature of the buffer $[(0.01 \mathrm{M}$ citrate buffer, $\mathrm{pH} 6.0$ ) at $95{ }^{\circ} \mathrm{C}$ ] and run in parallel with 
known positive and negative controls. After incubation with the primary antibody, relevant secondary antibodies were applied. Slides were then rinsed in water, soaked in phosphate-buffered saline and immunostained in a BioGenix I-6000 autostainer (San Ramon, CA, USA) using the biotin-streptavidin amplified system. Identical timing of incubations and washes was used for all cases. Positive controls included sections of adipose tissue for S14, normal heart for CD36, and placentafor FASN. Primary antibody was omitted and replaced with normal rabbit serum for negative control.

Staining interpretation Immunoreactivity for FASN, CD36, and S14 was interpreted without previous knowledge of any of the clinicopathological parameters. All slides were scored by one pathologist (PK). For each antibody, 20 randomly chosen cases were reviewed by a second pathologist to confirm reproducibility. For difficult cases, a consensus decision was made. Intraobserver reproducibility was established. The intensity and distribution of cytoplasmic (FASN and CD36) or nuclear staining (S14) were considered in the semiquantitative assessment of the immunohistochemical results. The intensity of cytoplasmic and nuclear staining was subjectively graded as negative, weak, and intense. For FASN, which is the focus of this study, we initially delineated positivity at $10 \%$ increments, but at 10-20\% cutoffs we were not able to establish reproducibility of grading. At a cutoff of $30 \%$, we achieved sustained reproducibility of grading and were able to separate positive and negative cases.

Only small number of cases showed completely negative staining in all tumor cells (4/86). Given that FASN is an ubiquitous enzyme, this was an expected finding. Cases that demonstrated intense staining of more than $30 \%$ of tumor cells were considered overexpression of the protein. The rest of the cases were considered low expression.

There was intra- and intercore heterogeneity: we observed variability of FASN staining in 15/86 cases. In tumors where, for example, cores 1 and 2 were graded as weak and core 3 as intense, using a $30 \%$ cutoff was relatively straightforward (if $>30 \%$ of the entire tumor is stained intense, overexpression was called). In occasional cases of intracore heterogeneity (where strongly staining cells were observed within a weak or negative background), the percent of intensely stained cells was averaged between the cores and if $>30 \%$ overexpression was assigned. Consensus was used in interpretation of these cases (see Supplement table 1). Thus, a cutoff of $30 \%$ (of the malignant cells) was chosen before the statistical analysis was undertaken. For consistency and for predictable inter- and intraobserver reproducibility the same cutoff was used for S14 and $\mathrm{CD} 36$

DLBCL cases were subtyped as germinal center (GC) vs nongerminal center (non-GC) according to the algorithm defined by Hans et al. [43], using antibodies against CD10 (PA0270, Leica Microsystems, IL, USA), MUM1 (PA0129, Leica Microsystems, IL, USA) and Bcl6 (PA0204, Leica Microsystems, IL, USA) as per manufacturer's instructions on Leica Bond III autostainer (Leica Microsystems, Newcastle, UK), using the bond polymer refine detection system (DS9800, Newcastle, UK). Staining was considered positive if $30 \%$ or more of the tumor cells stained for the antibody. The $30 \%$ cutoff for CD10, MUM1, and Bcl6 was based on published criteria for evaluation of TMA material [43].

Statistical analysis Univariate analysis of candidate prognostic marker association with overall survival for all cases, irrespective of rituximab treatment, was evaluated using chisquare tests $\left(\chi^{2}\right)$ [44]. A two-group $t$ statistic was used to measure the ability of a parameter to discriminate between the DLBCL subgroups. Survival and progression-free survival were estimated by the Kaplan-Meier method and compared by the log-rank test.

Survival and progression-free survival were fit to a multivariate Cox proportional hazard models $(\mathrm{CPH})$ stratified on the presence or absence of rituximab therapy (PROC PHREG, SAS v. 9.2, SAS Institute, Inc., Cary, NC, USA). The following variables were used in the multivariate: IPI (high, with low as reference), subtype (non-GC, unclassifiable, with GC as reference), FASN (overexpression, with low expression as reference), CD36 (overexpression, with low expression as reference), S14 (overexpression, with low expression as reference), and type (de novo, with transformed as reference).

Reduced models were built using stepwise backwards selection that removed noncontributory independent variables from the model when $p \geq 0.1$. Hazard ratios (HRs) with $95 \%$ confidence interval $(\mathrm{CI})$ were estimated, and fully adjusted survival curves were prepared from the $\mathrm{CPH}$ (Microsoft Office Excel 2007, Microsoft, Corp., Redmond, WA, USA). No adjustments were made for multiple hypothesis tests. The level of significance was set at $p \leq 0.05$.

\section{Results}

\section{Clinicopathological data}

The data set included 44 women and 42 men with mean age of $60 \pm 19.3$ years (Table 1). Of 86 patients, 35 died of the disease and 51 remained in complete remission after a median follow-up of 3.4 years (range, $0-15.5$ years). Sixty-seven cases $(78 \%$ ) presented with de novo lymphoma, and 19 cases were transformations of indolent lymphomas (9 follicular lymphoma, 2 chronic lymphocytic leukemia, 4 marginal zone lymphoma, 1 Hodgkin's lymphoma, and 3 indolent lymphomas not further characterized). 
Table 1 Patients' characteristics

\begin{tabular}{|c|c|c|}
\hline Characteristic & Number of patients & Percentage \\
\hline \multicolumn{3}{|l|}{ Gender } \\
\hline Male & 42 & 49 \\
\hline Female & 44 & 51 \\
\hline Age & $60.2 \pm 19.3$ & \\
\hline Age $>60$ & 46 & 53 \\
\hline Average follow-up & $4.2(0.1-15.5)$ & \\
\hline \multicolumn{3}{|l|}{ IPI } \\
\hline Low & 44 & 57 \\
\hline High & 33 & 43 \\
\hline \multicolumn{3}{|l|}{ Stage } \\
\hline I & 20 & 24 \\
\hline II & 19 & 22 \\
\hline III & 19 & 22 \\
\hline IV & 22 & 25 \\
\hline Not staged & 6 & 7 \\
\hline \multicolumn{3}{|l|}{ Subtype } \\
\hline $\mathrm{GC}$ & 39 & 45 \\
\hline Non-GC & 41 & 48 \\
\hline Unclassifiable & 6 & 7 \\
\hline \multicolumn{3}{|l|}{ Treatment } \\
\hline Not completed the 1 treatment & 14 & 16 \\
\hline 1 & 38 & 44 \\
\hline$>1$ & 34 & 40 \\
\hline
\end{tabular}

Thirty-five patients received standard cyclophosphamide, doxorubicin, vincristine, and prednisone (CHOP) treatment regime, and 52 patients received rituximab added to standard CHOP (R-CHOP). Thirty-nine cases were subtyped as GC type, 41 represented non-GC type, and 6 were unclassifiable. The IPI scores were available for 77 patients (44 high and 33 low IPI).

FASN, S14, and CD36 expression and correlation with mortality outcome and DLBCL subtype

The immunostaining pattern for FASN expression was cytoplasmic (Fig. 1), and its expression did not correlate with DLBCL subtype. FASN was overexpressed in $41 / 86$ cases (48\%) and correlated with poor outcome (dead vs alive) in univariate analysis. Twenty-four of $41(58 \%)$ patients with overexpression of FASN died (from either primary refractory disease or after multiple relapses, Table 2) compared to $11 / 45(25 \%)$ who did not overexpress FASN $(p=0.001)$. FASN overexpression correlated with decreased overall survival ( $p=0.002$, Fig. 2a). Similarly, the progression-free period was shorter in patients who exhibited overexpression of FASN ( $p=0.004$, Fig. $2 b$ ), and such patients were more likely to fail first-line therapy (Table 2).
S14 was expressed in the nuclei of the lymphoma cells (Fig. 1) and was overexpressed in 48/86 cases (55\%) (Table 2). It did not correlate with disease outcome (Table 2) or DLBCL subtype (GC and non-GC).

CD36 was expressed in the cytoplasm and was overexpressed in 61/86 (70\%) cases (Fig. 1). Overexpression of CD36 as a single risk factor did not correlate with outcome (Table 2), but predicted a non-GC DLBCL subtype ( $p=$ $0.017)$. We found no correlation between expression of FASN, S14, or CD36.

FASN, Spot14, and CD36 are independent predictors of overall survival

In a multivariate analysis, patients were stratified depending on whether they received rituximab with $\mathrm{CHOP}$ chemotherapy. In the group treated with R-CHOP, high IPI $[\mathrm{HP}=4.2$ (CI $1.302-17.027), p=0.025]$ and overexpression of FASN $[\mathrm{HP}=$ 4.09 (CI 1.287-16.04), $p=0.025$ ] were significant risk factors, and overexpression of $\mathrm{CD} 36$ was a protective factor $[\mathrm{HR}=$ 0.34 (CI 0.12-0.96), $p=0.037$ ]. Similar results were found in $\mathrm{R}-\mathrm{CHOP}$ patients in regards to progression-free survival. High IPI [HR=8.61 (CI 2.82-30.937), $p=0.0004]$, overexpression of FASN $[\mathrm{HR}=4.20$ (CI 1.542-12.371), $p=0.006]$ were significant risk factors, and overexpression of $\mathrm{CD} 36$ was a protective factor $[\mathrm{HR}=0.218$ (CI 0.061-0.698), $p=0.013$ ]. In patients treated with CHOP alone, high IPI $(p=0.004)$, but not FASN, was an independent predictor of poor survival.

To further elucidate the prognostic significance of CD36 in a cohort of patients treated with R-CHOP, we calculated survival distribution estimates from the $\mathrm{CPH}$-reduced models fully adjusted for CD36 levels (see "Methods"). Intriguingly, CD36 overexpression improved survival in patients independent of IPI score and FASN expression (Fig. 3). As expected, patients with low IPI and low FASN expression exhibited improved overall and progression-free survival. Both survival measures deteriorated with either high IPI or FASN overexpression. FASN overexpression further refined prognosis assessed based on IPI (Fig. 3).

\section{Discussion}

We analyzed a cohort of 86 patients for the prognostic significance of FASN, S14, and CD36 expression in DLBCL. Most normal tissue cells, even those with high cell turnover, preferentially use extracellular circulating lipids for the source of intracellular structural lipids [10, 47]. By contrast, a wide variety of tumors and their precursor lesions undergo augmented de novo biogenesis of FAs irrespective of the levels of circulating lipids [8]. Neoplastic lipogenesis is accompanied by significantly increased expression and activity of several lipogenic enzymes in tumor cells. Among these, upregulation 
Fig. 1 Representative cytoplasmic low expression (a) and overexpression (b) of FASN, magnification $\times 400$. Representative nuclear low expression (c) and overexpression (d) of S14, magnification $\times 400$. Representative cytoplasmic low expression (e) and overexpression (f) of CD36, magnification $\times 400$
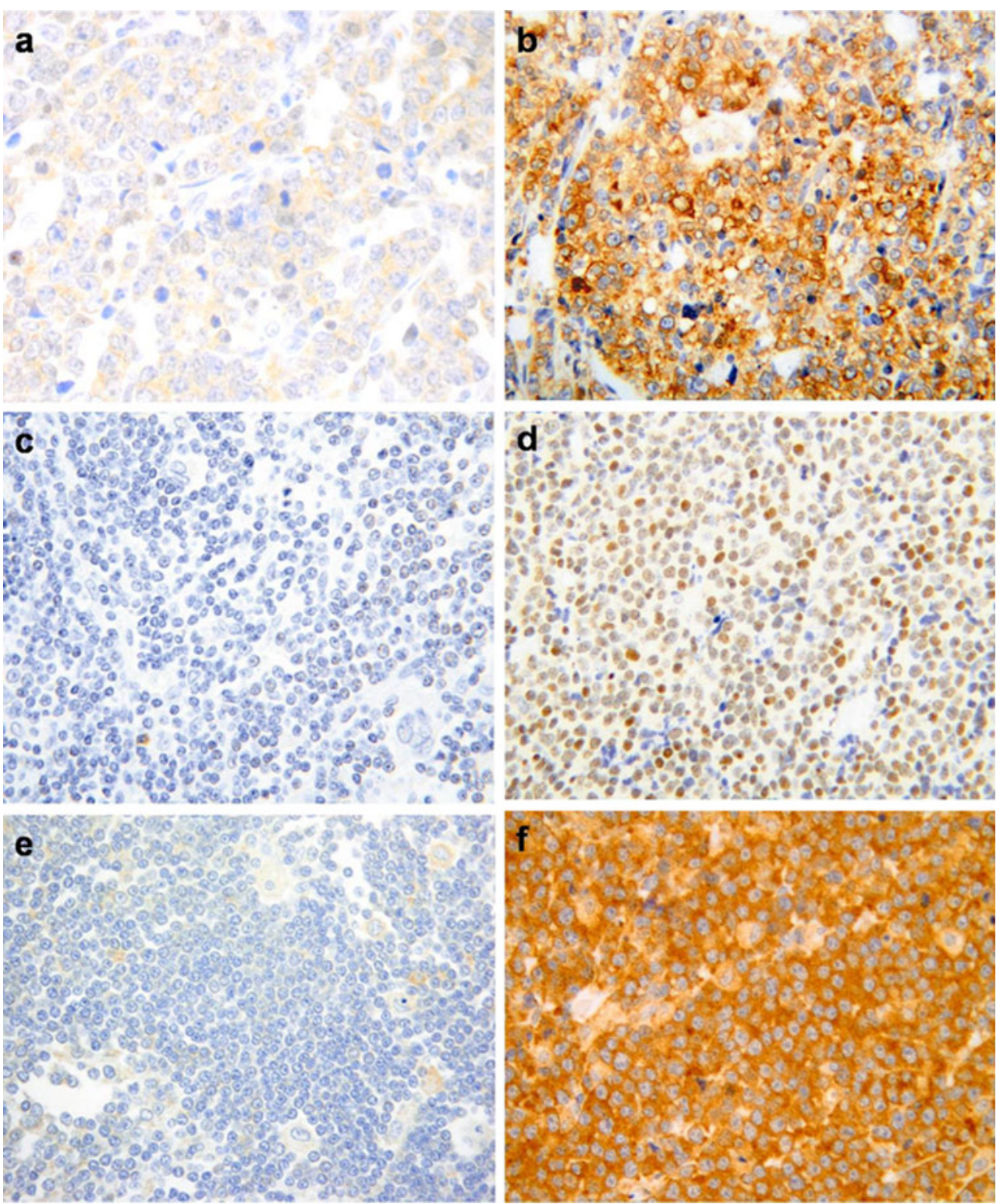

of FASN represents a nearly universal phenotypic alteration in most human malignancies [10, 23, 48].

There are limited data on the role of lipogenic proteins in non-Hodgkin lymphoma in general and DLBCL in particular. In this study, we demonstrate that overexpression of
FASN is an independent predictor of decreased overall and progression-free survival in patients with DLBCL. This finding suggests that overexpression of FASN and thus enhanced lipid metabolism contribute to a more aggressive lymphoma phenotype with worse prognosis similarly to that

Table 2 Association of prognostic markers with overall survival and number of treatment regimens

\begin{tabular}{|c|c|c|c|c|c|c|c|c|c|c|c|c|c|c|c|}
\hline & \multicolumn{2}{|c|}{ Subtype } & \multirow{2}{*}{$\frac{p \text { value }}{\chi^{2}}$} & \multicolumn{2}{|l|}{ IPI } & \multirow{2}{*}{$\frac{p \text { value }}{\chi^{2}}$} & \multicolumn{2}{|c|}{ FASN } & \multirow{2}{*}{$\frac{p \text { value }}{\chi^{2}}$} & \multicolumn{2}{|l|}{ S14 } & \multirow{2}{*}{$\frac{p \text { value }}{\chi^{2}}$} & \multicolumn{2}{|c|}{ CD36 } & \multirow{2}{*}{$\frac{p \text { value }}{\chi^{2}}$} \\
\hline $\begin{array}{l}\text { Overall Survival } \\
\qquad(n=86)\end{array}$ & $\mathrm{GC}$ & Non-GC & & $\mathrm{L}$ & $\mathrm{H}$ & & Low & Overexpression & & Low & Overexpression & & Low & Overexpression & \\
\hline $\begin{array}{l}\text { Complete } \\
\text { Remission }\end{array}$ & 24 & 24 & $p=0.784$ & 38 & 10 & $p=5.1 \mathrm{e}-7$ & 34 & 17 & $p=0.001$ & 22 & 29 & $p=0.812$ & 15 & 36 & $p=0.933$ \\
\hline Death & 15 & 17 & & 6 & 23 & & 11 & 24 & & 16 & 19 & & 10 & 25 & \\
\hline \multicolumn{16}{|c|}{ Number of treatments } \\
\hline 1 & 20 & 17 & $p=0.456$ & 31 & 6 & $p=0.0003$ & 28 & 10 & $p=0.003$ & 16 & 22 & $p=0.938$ & 12 & 26 & $p=0.739$ \\
\hline$>1$ & 13 & 16 & & 12 & 17 & & 13 & 21 & & 14 & 20 & & 12 & 22 & \\
\hline
\end{tabular}

$L$ low IPI, $H$ high IPI 


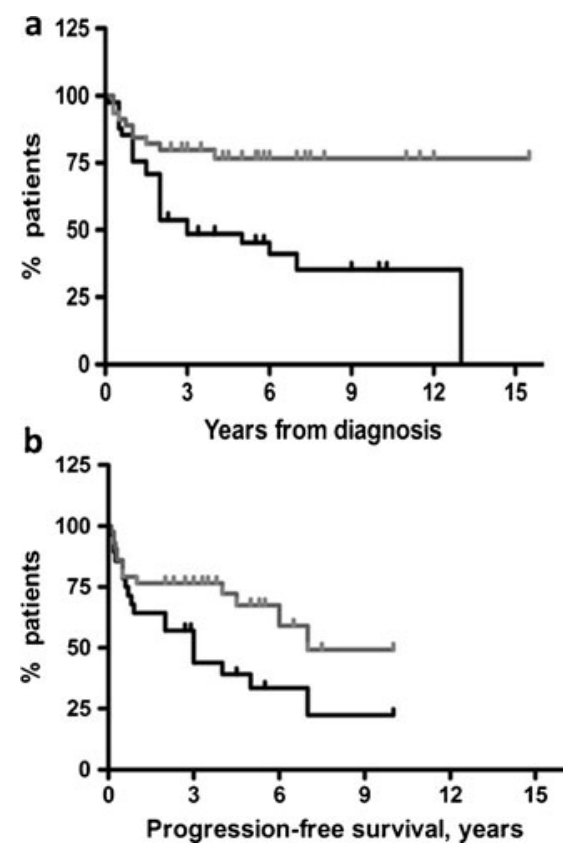

Fig. 2 a Kaplan-Meier plot of survival (time period from the diagnosis to the endpoint) for low expression (gray line) and overexpression (black line) of FASN ( $p=0.002, \mathrm{HR}=2.92,95 \%$ CI 1.180-5.803). b Kaplan-Meier plot of progression-free survival for low expression and overexpression of FASN ( $p=0.004, \mathrm{HR}=3.96,95 \%$ CI $1.547-10.45)$. Notch denotes the censored data

reported in other malignancies $[17,18]$. Our results differ from those reported by Uddin et al. [49], who demonstrated no prognostic significance of FASN expression in primary DLBCL tissues. This may be due to differences in the scoring system. We used a $30 \%$ cutoff and strong positivity for delineation of overexpression, with $48 \%$ of cases classified as positive, whereas in the latter work, use of an " $\mathrm{H}$ score" resulted in a higher number of positive cases $(62.6 \%)$. Our finding of the prognostic significance of FASN corroborates reports of a correlation between FASN expression levels and the established markers of tumor aggressiveness, such as $\mathrm{Ki}-67$ and $\mathrm{p}-\mathrm{AKT}$, in DLBCL
[49]. Furthermore, pharmacologic and small interfering RNA-mediated knockdown of FASN-induced apoptosis in DLBCL and mantle cell lymphoma cell lines [49, 50].

In this study, IPI score remained a reliable prognostic marker [45, 46], while DLBCL subtype did not predict survival. The latter can perhaps be explained by the addition of rituximab to the standard therapy in the late 1990s [51-53].

Interestingly, FASN expression was not associated with that of S14, which suggests that FASN may be regulated in an S14-independent manner in the lymphoma cells. CD36 expression also did not correlate with FASN expression, suggesting that de novo FA synthesis may exist in the malignant cells regardless of potential lipid uptake by the cells.

FASN expression pattern is an independent prognostic marker in patients with DLBCL treated with R-CHOP. Poor prognosis in patients with high IPI who overexpressed FASN and relatively worsened prognosis in patients with low IPI who still had high FASN expression further supports the notion that "lipogenic phenotype" contributes to disease aggressiveness in DLBCL.

Overexpression of CD36 was protective and improved both overall and progression-free survival in patients treated with R-CHOP. CD36 was seen only in the cytoplasm, which suggests defects in hormonal pathways involved in trafficking of the molecule to the cell surface. CD36 has multiple functions including that of a thrombospondin receptor [34, 35]. The thrombospondin family of proteins is important angiogenesis inhibitors. It has been recently shown that a recombinant fragment of thrombospondin-2 inhibits breast cancer growth and metastasis through CD36-mediated activation of endothelial cell apoptosis [54]. One may argue that CD36 expression may be associated with increased antiangiogenic and proapoptotic activity in lymphoma cells with less aggressive phenotype. In addition, rituximab targets the transmembrane protein CD20 and may directly induce apoptosis. Thus, the lipid membrane composition may be an important factor for the activity of rituximab and may be mediated by CD36.
Fig. 3 Multivariate survival solution of overall survival with rituximab therapy. The $\mathrm{CPH}$ survival solution when patients are treated with rituximab is stratified by CD36 expression, R-IPI scoring, and FASN expression. The classic R-IPI scoring prognostic value is seen with low scores predicting longer survival. Low FASN expression and CD36 overexpression also predict a longer survival. $L$ low, $H$ high, $O$ overexpression
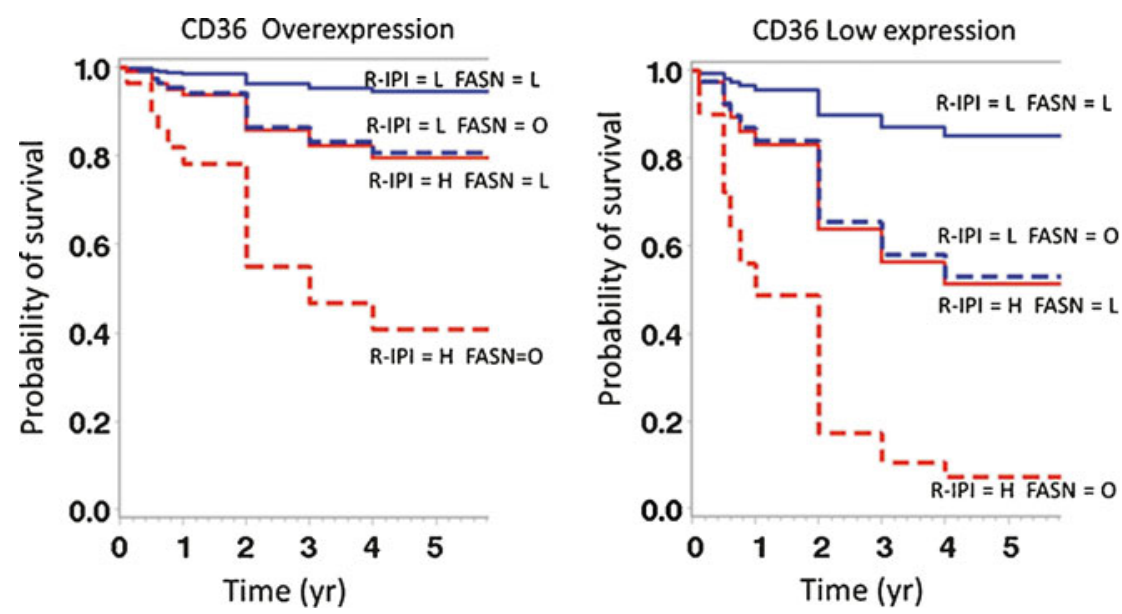
The limitations of this study include a need for validation of the pattern obtained in TMA compared to whole slides to account for inhomogeneous expression. Additionally, we believe that fluorescent in situ hybridization analysis to account for prognostic significance of Myc gene rearrangements in DLBCL treated with R-CHOP would be of value. Furthermore, we recognize that our results need to be validated in a larger study involving multiple institutions.

In summary, we demonstrate that FASN and CD36 overexpression may serve as independent prognostic markers in DLBCL.

FASN may represent potential therapeutic target in DLBCL. Thus, further studies to investigate mechanistic links between FA synthesis and uptake with clinical parameters, molecular, and transcriptional alterations in DLBCL are warranted.

Acknowledgments The authors sincerely thank Vincent A. Memoli, $\mathrm{MD}$, for his technical expertise and assistance with interpretation of immunohistochemical staining and Nancy Kuemmerle, MD, for providing the S14 antibody. Funding acknowledgements: NIH grant RO1CA126618 (W.K.)

Disclaimers/conflict of interest The authors declare that they have no conflict of interest.

\section{References}

1. Anderson JR, Armitage JO, Weisenburger DD (1998) Epidemiology of the non-Hodgkin's lymphomas: distributions of the major subtypes differ by geographic locations. Non-Hodgkin's lymphoma classification project. Ann Oncol 9:717-720

2. Swerdlow SH, Campo E, Harris NL, Jaffe ES, Pileri SA, Stein H, Thiele J, Vardiman JW (2008) WHO classification of tumours of haematopoietic and lymphoid tissues, 4th edn. International Agency for Research on Cancer, Lyon

3. Gatenby RA, Gillies RJ (2004) Why do cancers have high aerobic glycolysis? Nat Rev Cancer 4:891-899

4. Swinnen JV, Beckers A, Brusselmans K et al (2005) Mimicry of a cellular low energy status blocks tumor cell anabolism and suppresses the malignant phenotype. Cancer Res 65:2441-2448

5. Warburg O (1956) On the origin of cancer cells. Science 123:309-314

6. Clemens MJ (2004) Targets and mechanisms for the regulation of translation in malignant transformation. Oncogene 23:3180-3188

7. Rahman L, Voeller D, Rahman M et al (2004) Thymidylate synthase as an oncogene: a novel role for an essential DNA synthesis enzyme. Cancer Cell 5:341-351

8. Medes G, Thomas A, Weinhouse S (1953) Metabolism of neoplastic tissue. IV. A study of lipid synthesis in neoplastic tissue slices in vitro. Cancer Res 13:27-29

9. Kuhajda FP (2000) Fatty-acid synthase and human cancer: new perspectives on its role in tumor biology. Nutrition 16:202-208

10. Menendez JA, Lupu R (2007) Fatty acid synthase and the lipogenic phenotype in cancer pathogenesis. Nat Rev Cancer 7:763-777

11. Asturias FJ, Chadick JZ, Cheung IK et al (2005) Structure and molecular organization of mammalian fatty acid synthase. Nat StructMolBiol 12:225-232

12. Wakil SJ (1989) Fatty acid synthase, a proficient multifunctional enzyme. Biochemistry 28:4523-4530
13. Pizer ES, Jackisch C, Wood FD et al (1996) Inhibition of fatty acid synthesis induces programmed cell death in human breast cancer cells. Cancer Res 56:2745-2747

14. Wang WQ, Zhao XY, Wang HY et al (2008) Increased fatty acid synthase as a potential therapeutic target in multiple myeloma. $\mathrm{J}$ Zhejiang UnivSci B 9:441-447

15. Milgraum LZ, Witters LA, Pasternack GR et al (1997) Enzymes of the fatty acid synthesis pathway are highly expressed in in situ breast carcinoma. Clin Cancer Res 3:2115-2120

16. Brusselmans K, De Schrijver E, Verhoeven G et al (2005) RNA interference-mediated silencing of the acetyl-CoA-carboxylase-alpha gene induces growth inhibition and apoptosis of prostate cancer cells. Cancer Res 65:6719-6725

17. Alo PL, Visca P, Marci A et al (1996) Expression of fatty acid synthase (FAS) as a predictor of recurrence in stage I breast carcinoma patients. Cancer 77:474-482

18. Gansler TS, Hardman W 3rd, Hunt DA et al (1997) Increased expression of fatty acid synthase (OA-519) in ovarian neoplasms predicts shorter survival. Hum Pathol 28:686-692

19. Alo PL, Visca P, Trombetta G et al (1999) Fatty acid synthase (FAS) predictive strength in poorly differentiated early breast carcinomas. Tumori 85:35-40

20. Rashid A, Pizer ES, Moga M et al (1997) Elevated expression of fatty acid synthase and fatty acid synthetic activity in colorectal neoplasia. Am J Pathol 150:201-208

21. Kuhajda FP (2006) Fatty acid synthase and cancer: new application of an old pathway. Cancer Res 66:5977-5980

22. Pizer ES, Wood FD, Heine HS et al (1996) Inhibition of fatty acid synthesis delays disease progression in a xenograft model of ovarian cancer. Cancer Res 56:1189-1193

23. Kuhajda FP, Jenner K, Wood FD et al (1994) Fatty acid synthesis: a potential selective target for antineoplastic therapy. Proc Natl Acad Sci USA 91:6379-6383

24. Kuhajda FP, Pizer ES, Li JN et al (2000) Synthesis and antitumor activity of an inhibitor of fatty acid synthase. ProcNatlAcadSci USA 97:3450-3454

25. Cunningham BA, Moncur JT, Huntington JT et al (1998) "Spot 14" protein: a metabolic integrator in normal and neoplastic cells. Thyroid 8:815-825

26. Jump DB, Oppenheimer JH (1985) High basal expression and 3,5,3'-triiodothyronine regulation of messenger ribonucleic acid S14 in lipogenic tissues. Endocrinology 117:2259-2266

27. Kinlaw WB, Quinn JL, Wells WA et al (2006) Spot 14: a marker of aggressive breast cancer and a potential therapeutic target. Endocrinology 147:4048-4055

28. Wells WA, Schwartz GN, Morganelli PM et al (2006) Expression of "Spot 14" (THRSP) predicts disease free survival in invasive breast cancer: immunohistochemical analysis of a new molecular marker. Breast Cancer Res Treat 98:231-240

29. Donnelly C, Olsen AM, Lewis LD et al (2009) Conjugated linoleic acid (CLA) inhibits expression of the Spot 14 (THRSP) and fatty acid synthase genes and impairs the growth of human breast cancer and liposarcoma cells. Nutr Cancer 61:114-122

30. Kinlaw WB, Church JL, Harmon J et al (1995) Direct evidence for a role of the "spot 14" protein in the regulation of lipid synthesis. J Biol Chem 270:16615-16618

31. Brown SB, Maloney M, Kinlaw WB (1997) "Spot 14" protein functions at the pretranslational level in the regulation of hepatic metabolism by thyroid hormone and glucose. J Biol Chem 272:2163-2166

32. Breuker C, Moreau A, Lakhal L et al (2010) Hepatic expression of thyroid hormone-responsive spot 14 protein is regulated by constitutive androstane receptor (NR1I3). Endocrinology 151:1653-1661

33. Martel PM, Bingham CM, McGraw CJ et al (2006) S14 protein in breast cancer cells: direct evidence of regulation by SREBP-1c, superinduction with progestin, and effects on cell growth. Exp Cell Res 312:278-288 
34. Kennedy DJ, Kashyap SR (2011) Pathogenic role of scavenger receptor CD36 in the metabolic syndrome and diabetes. MetabSyndrRelatDisord 9:239-245

35. Love-Gregory L, Abumrad NA (2011) CD36 genetics and the metabolic complications of obesity. CurrOpinClinNutrMetab Care 14:527-534

36. Febbraio M, Hajjar DP, Silverstein RL (2001) CD36: a class B scavenger receptor involved in angiogenesis, atherosclerosis, inflammation, and lipid metabolism. J Clin Invest 108:785-791

37. Kuemmerle NB, Rysman E, Lombardo PS et al (2011) Lipoprotein lipase links dietary fat to solid tumor cell proliferation. Mol Cancer Ther 10:427-436

38. Goldberg IJ, Eckel RH, Abumrad NA (2009) Regulation of fatty acid uptake into tissues: lipoprotein lipase- and CD36-mediated pathways. J Lipid Res 50:S86-S90

39. Ueno S, Yamaguchi M, Kimura M et al (2005) Expression of CD29 on lymphoma cells and/or CD36 on microvascular endothels correlates with high serum LDH level in diffuse large B-cell lymphomas (DLBCLs) and is frequent in de novo CD5-positive DLBCLs. Int J Oncol 27:1241-1246

40. Rutella S, Rumi C, Puggioni P et al (1999) Expression of thrombospondin receptor (CD36) in B-cell chronic lymphocytic leukemia as an indicator of tumor cell dissemination. Haematologica $84: 419-424$

41. A predictive model for aggressive non-Hodgkin's lymphoma. The International Non-Hodgkin's Lymphoma Prognostic Factors Project (1993) N Engl J Med 329:987-994

42. Sehn LH, Berry B, Chhanabhai M et al (2007) The revised International Prognostic Index (R-IPI) is a better predictor of outcome than the standard IPI for patients with diffuse large B-cell lymphoma treated with R-CHOP. Blood 109:1857-1861

43. Hans CP, Weisenburger DD, Greiner TC et al (2004) Confirmation of the molecular classification of diffuse large B-cell lymphoma by immunohistochemistry using a tissue microarray. Blood 103:275-282

44. Preacher KJ (2001) Calculation for the chi-square test: an interactive calculation tool for chi-square tests of goodness of fit and independence (Computer software). http://quantpsy.org
45. Ziepert M, Hasenclever D, Kuhnt E et al (2010) Standard International prognostic index remains a valid predictor of outcome for patients with aggressive CD20+ B-cell lymphoma in the rituximab era. J ClinOncol 28:2373-2380

46. Westin JR, Fayad LE (2009) Beyond R-CHOP and the IPI in largecell lymphoma: molecular markers as an opportunity for stratification. Curr Hematol Malig Rep 4(4):218-224

47. Weiss L, Hoffmann GE, Schreiber R et al (1986) Fatty-acid biosynthesis in man, a pathway of minor importance. Purification, optimal assay conditions, and organ distribution of fatty-acid synthase. Biol Chem Hoppe Seyler 367:905-912

48. Menendez JA, Lupu R (2004) Fatty acid synthase-catalyzed de novo fatty acid biosynthesis: from anabolic-energy-storage pathway in normal tissues to jack-of-all-trades in cancer cells. Arch ImmunolTherExp (Warsz) 52:414-426

49. Uddin S, Hussain AR, Ahmed M et al (2010) Inhibition of fatty acid synthase suppresses c-Met receptor kinase and induces apoptosis in diffuse large B-cell lymphoma. Mol Cancer Ther 9 (5):1244-1255

50. Gelebart P, Zak Z, Anand M et al (2912) Blockade of fatty acid synthase triggers significant apoptosis in mantle cell lymphoma. PLoS One 7(4):e33738

51. Sehn LH, Donaldson J, Chhanabhai M et al (2005) Introduction of combined CHOP plus rituximab therapy dramatically improved outcome of diffuse large B-cell lymphoma in British Columbia. J ClinOncol 23(22):5027-5033

52. Fu K, Weisenburger DD, Choi WW et al (2008) Addition of rituximab to standard chemotherapy improves the survival of both the germinal center B-cell-like and non-germinal center B-cell-like subtypes of diffuse large B-cell lymphoma. J ClinOncol 26:4587-4594

53. van Krieken JH (2009) New developments in the pathology of malignant lymphoma: a review of the literature published from January to August 2009. J Hematop 2:171-183

54. Koch M, Hussein F, Woeste A et al (2011) CD36-mediated activation of endothelial cell apoptosis by an $\mathrm{N}$-terminal recombinant fragment of thrombospondin-2 inhibits breast cancer growth and metastasis in vivo. Breast Cancer Res Treat 128:337-346 